\title{
Safety Information Modeling: Smart Safety Devices \& Internet of Everything
}

\author{
S B Aanandh \\ Honeywell Technology Solutions, Bangalore, 560076, India \\ Email: s.b.aanandh@gmail.com \\ Chinmaya Kar ${ }^{1}$, Nihal Siddiqqui ${ }^{2}$ \\ ${ }^{1}$ Honeywell Technology Solutions, Bangalore, 560076, India \\ ${ }^{2}$ Univ. Petroleum \& Energy Studies, Dehradun, Uttarakhand \\ Email: Chinmaya.kar@gmail.com, nsiddiqui57@gmail.com
}

\begin{abstract}
The next generation of Internet of things that connects the things, people and the process through which the people and things interact is coined as Internet of Everything. Safety management is constructed as a complex system of systems design coordinating with each other like the Fire Alarm System or Gas Detection System as well as the Emergency response like the Fire Fighters and Para-Medicals like the Ambulatory services. The governments have been setting up national broadband plans and separate dedicated spectrum for Public Safety Communications used for effective information rich emergency management and response. This paper outlines the evolution of the public safety LTE network and its applicability in the safety management system and safety preparedness. It also describes the role of Smart Objects and Internet of Everything in Safety Management. To achieve this, this paper develops the information models for safety management systems to be used in IoE utilizing the broad-band LTE networks.
\end{abstract}

Index Terms - Safety Management Systems, Information Modeling, Internet of Everything, Public Safety LTE.

\section{INTRODUCTION}

Internet of Things (IoT) is widely known and typically brought to the fore in the context of Ma-chine-toMachine (M2M) communications. IOT evolution has given rise to the genesis of new subject called Internet of Everything (IoE). IoE connects the products, the users(people) and the process through which these interact. IoE has been a subject of debate for many applications such as improving operation effectiveness in a business, to increased safety or better management of urban facilities and smarter cities [1].The concept of IoE is piloted by Communications Company such as Cisco or Qualcomm. IoE thus is a heterogeneous connection of smart-products with consumers (users) and the process in which these are operated.

Safety management on the other hand can be defined as a businesslike approach to safety. It is a systematic, explicit and comprehensive process for managing safety risks. As with all management systems, a Safety Management System(SMS) provides for goal setting, planning, and measuring performance. A SMS is woven into the fabric of an organization. It becomes part of the culture and is the way people do their jobs [2]. Globally, governments have begun to adopt a national broadband plan and also provide a dedicated spectrum for Public Safety using the Evolved Packet Core Long term Evolution.

In this paper we describe how the new media of $\mathrm{IoE}$, the LTE may facilitate the increased system-safety and deduce the information models for effective safety management systems. We take inspiration from Building information modeling [3] that has steadily grown and captured the minds of Architects, builders and operators alike to deduce the Safety Information Model.

Safety management deals with both the prevention of accidents and as well as managing emergencies [4].The suitability of the LTE networks and the architectures for emergency response has been detailed out by the [5]. Safety Life Cycle encompasses design corrections, periodic maintenance, layers of protection to emergency management; this paper derives the information models useful for safety management.

\section{Public Safety Communications: 3GPP LTE}

Public safety networks provide communications for services such as police, fire and ambulance. In this realm the requirement has been to develop systems that are highly robust and can address the specific communication needs of emergency services. This has fostered public safety standards - such as TETRA and P25 - that provide a set of features that were not previously supported in commercial cellular systems. These standards have also been applied to commercial critical communications needs such as airport operations or Industrial Management.

The shortcoming of these narrowband applications is their insufficient bandwidths for high data rate applications i.e. file transfers and streaming media. [6]. Initially the thought to adapt nationwide broadband communications originated in the United States around late 2009 and slowly different countries across the globe have begun to adopt a similar thought. The availability of high data throughput to these essential services not only 
helps them in informed decision-ing but also in evolution of applications that are integral to safety Management.

The TETRA and P25 systems on the other hand provided specific communication access methods like the Push-To-Talk(PTT), ease of deployment with minimal central controller(network router). These features are critical for emergency communications. The 3GPP group is adding the following specifications as part of release 12 and release 13 to meet these specific needs.

Table 1. 3GPP Evolution [7]

\begin{tabular}{ccc}
\hline Work Item & 3GPP Release & Work Item Document Reference \\
\hline Proximity-based Services Specification (ProSe) & 12 & SP-130030 [5] \\
Group Communication System Enablers for LTE (GCSE_LTE) & 12 & SP-130326 [6] \\
\hline Public Safety Broadband High Power User Equipment for Band 14 for Region 2 & 11 & RP-120362 [7] \\
\hline Study on Resilient E-UTRAN Operation for Public Safety (FS_REOPS) & 12 & SP-130240 [8] \\
\hline
\end{tabular}

Texas A\&M University \& the EDGE Innovation network are also working on Safety LTE evolution and the advancement of associated telemetry equipments, mobile handsets and situational awareness applications. [7].

\section{SAFETY MANAGEMENT AND SAFETY PREPAREDNESS}

A safety management system provides a systematic way to identify hazards and control risks while maintaining assurance that these risk controls are effective. SMS can be defined as: a businesslike approach to safety. It is a systematic, explicit and comprehensive process for managing safety risks. As with all management systems, a safety management system provides for goal setting, planning, and measuring performance. A safety management system is woven into the fabric of an organization. It becomes part of the culture, the way people do their jobs [8].

In general Safety is defined in terms of identified hazards (HAZID) and achieving lower Risk levels as in ALARP “As Low $\boldsymbol{A}$ s $\boldsymbol{R}$ easonably Practicable".

Dr. Wayne Blanchard and Dr. Cortez Lawrence in 2007 set up a working group for defining the principles of Emergency Management \& Disaster Preparedness and they developed the eight principles presented in Table 2

Table 2. Principles of Emergency management

\begin{tabular}{|c|c|c|}
\hline \# & Principle & Description \\
\hline 1. & Comprehensive & $\begin{array}{l}\text { Emergency managers consider and take into account all hazards, } \\
\text { all phases, all stakeholders and all impacts relevant to disasters. }\end{array}$ \\
\hline 2. & Progressive & $\begin{array}{l}\text { Emergency managers anticipate future disasters and take preventive and preparatory measures } \\
\text { to build disaster-resistant and disaster-resilient communities. }\end{array}$ \\
\hline 3. & Risk-driven & $\begin{array}{l}\text { Emergency managers use sound risk management principles } \\
\text { (hazard identification, risk analysis, and impact analysis) in assigning priorities and resources. }\end{array}$ \\
\hline 4. & Integrated & Emergency managers ensure unity of effort among all levels of government and all elements of a community. \\
\hline 5. & Collaborative & $\begin{array}{l}\text { Emergency managers create and sustain broad and sincere relationships among individuals and organizations } \\
\text { to encourage trust, advocate a team atmosphere, build consensus, and facilitate communication. }\end{array}$ \\
\hline 6. & Coordinated & Emergency managers synchronize the activities of all relevant stakeholders to achieve a common purpose. \\
\hline 7. & Flexible & Emergency managers use creative and innovative approaches in solving disaster challenges. \\
\hline 8. & Professional & $\begin{array}{l}\text { Emergency managers value a science and knowledge-based approach; based on education, } \\
\text { training, experience, ethical practice, public stewardship and continuous improvement. }\end{array}$ \\
\hline
\end{tabular}

Emergency management is a subset in the overall safety life cycle as in Safety management and both are constructed by design and expect risk reduction by clear objectives, continuous monitoring and collaboration amongst different entities. Internet of things is evolving and getting applicability in multiple domains, and as Internet has become a pervasive way of collaboration, Safety and Emergency management would drive on the new era of Internet of Things or Internet of Everything.

\section{INTERNET OF EVERYTHING \& SMART OBJECTS}

Smart Objects are things that can communicate with users and other things about possible interactions with itself. The Auto-ID project in MIT Labs conceived these things as devices equipped with RFID tags and one can know more information by querying these devices. With the rise of Ubiquitous and pervasive networking, the concept grew to a level that the devices communicate with each other over Internet and also connect to god-like data banks i.e. the cloud computers. Several methodologies are being developed to make internet of things being a reality. These include CoAP (Constrained Applications Protocol) similar to SOAP services, MQTT (Message Queuing Transport Telemetry) and RESTful http services. (Dinh \& Kim, 2012).In early 2013 Cisco \& Qualcomm began discussing about Internet of Everything and the definition provided by Qualcomm was 
"The Internet of Everything is based on the idea that everyday objects can be readable, recognizable, locatable, addressable, and controllable via the Internet. Although the market defines the Internet of Everything in terms of connected everyday objects, the nature of the connection remains to be determined."

Cisco on the other hand defined the Internet of Everything (IoE) as "bringing together people, process, data, and things to make networked connections more relevant and valuable than ever before-turning information into actions that create new capabilities, richer experiences, and unprecedented economic opportunity for businesses, individuals, and countries".

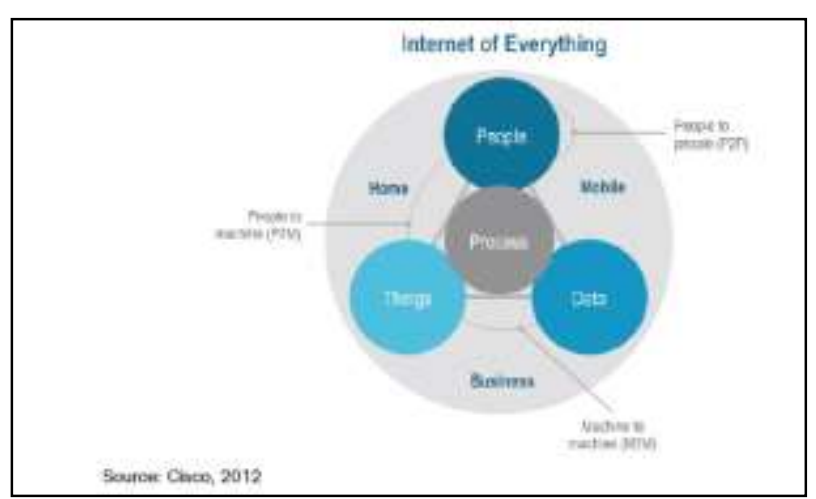

Internet of Everything finds application in different areas i.e. Energy Management \& Smart Grid, Manufacturing and Retail and the concept of Internet of Everything is being pursued in building smarter cities from Songdo (South Korea), Nice (France) etc. The aspect of connecting devices and people has been widely utilized in more commercial and comforting applications. The aspect of safety governance is not yet been widely studied and as noted earlier the Safety communications have been in specific networks like TETRA. The connection of the safety applications with the Internet is evolving with the broadband wireless initiatives.

The CAPSCOM program of California Public Safety [9] outlines the need of the Broadband-ing work to evolve as a Systems-of-Systems solutions rather than a single System solution. In this context the IoE solution fits well as a set of coherently defined federated services. The systems-of-systems solutions require different systems to communicate seamlessly which necessitates building the semantics of the information and modeling it. In this paper we take up this case and devise the information model for the safety systems or in-short Safety Information Model.

Fig. 1. Internet of Everything (IoE) [15]

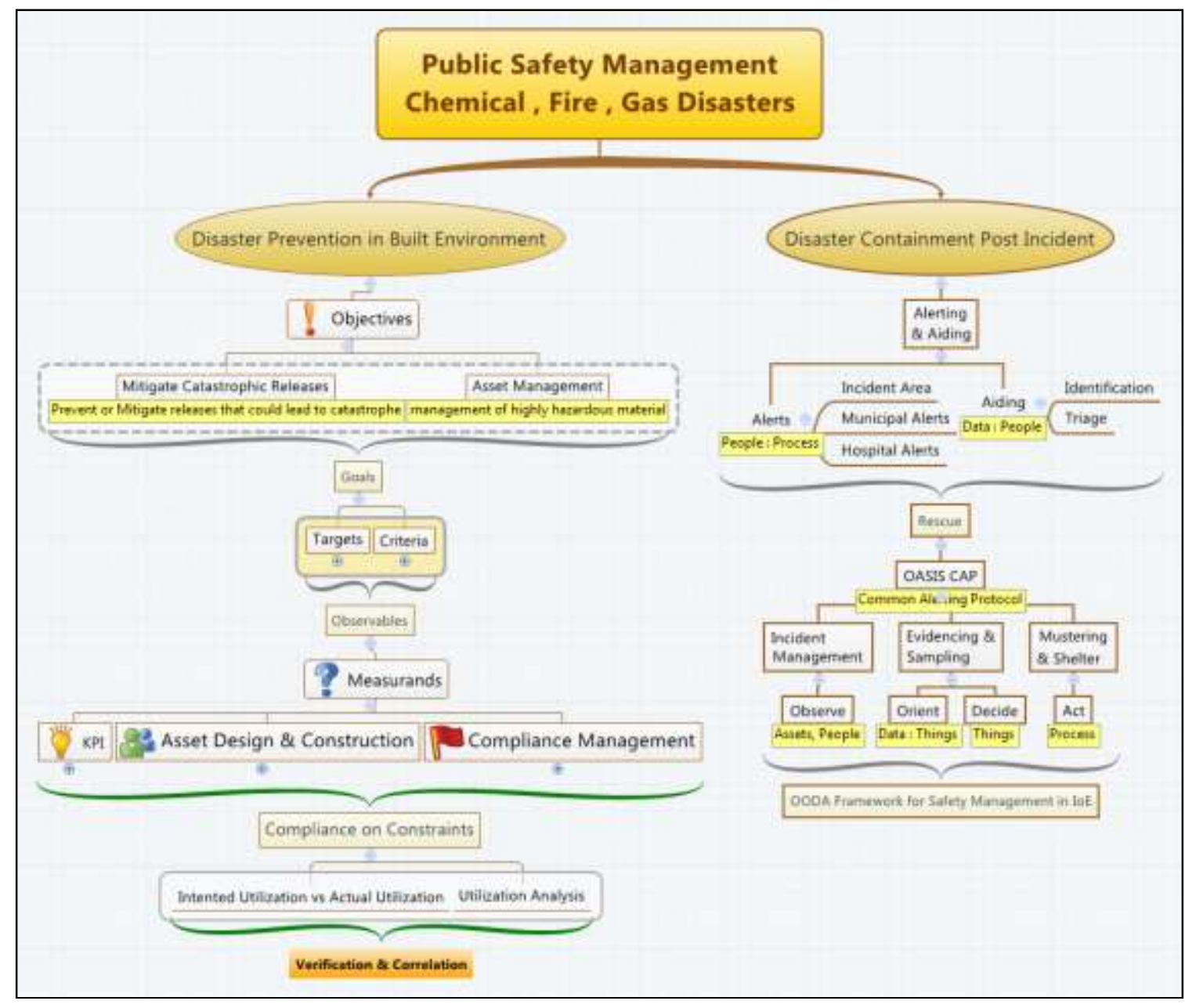

Fig. 2. Safety Information Model Overview 


\section{INFORMATION MODELING FOR SAFETY MANAGEMENT $\&$ COMPLIANCE}

As discussed earlier, Safety management provides a systematic way to reduce the risk levels to as lowest as possible in theory. The effectiveness of a safety management system is heavily dependent on the practice maturity, compliance to standards adherence and continuous monitoring. The Public Safety LTE global research is geared up towards emergency management that is one element of the Safety management System [5]. The disaster preparedness and control requires information that is regularly sampled and about compliance adherence. The evolution of the information model is represented as a mind-map with information labels representing the Nodes role in the IoE context
(People, Data or Process). For the study purpose, the scope was restricted to Fire \& Gas Hazard Management.

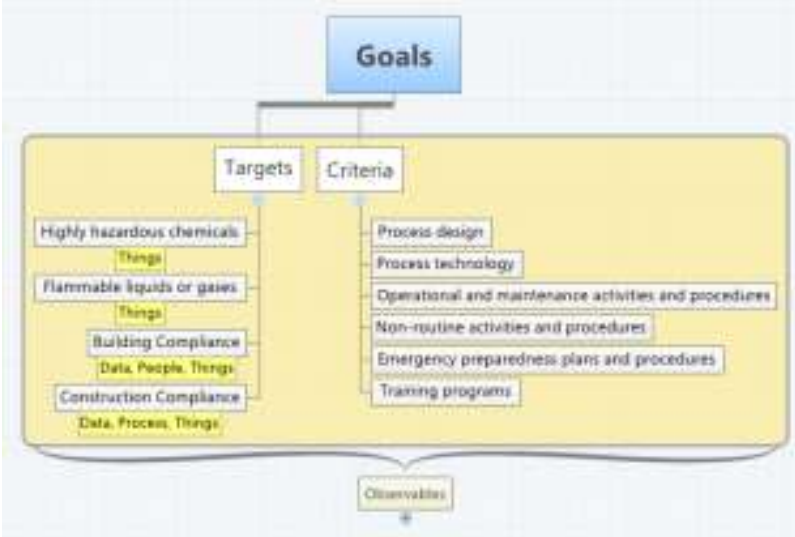

Fig. 3. Goals for Safety management for Disaster Mitigation

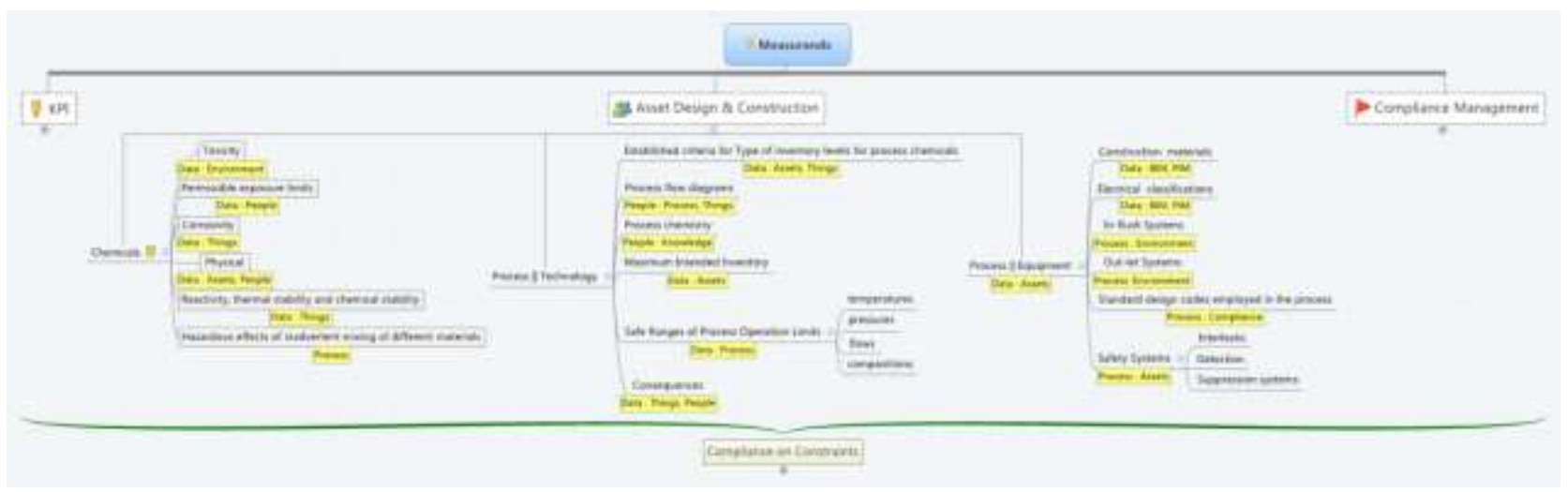

Fig. 4. Asset Design \& Construction

The overall Disaster Management takes into account both the conditions, i.e. the mitigation planning and the disaster containment after an incident as occurred i.e. Disaster prevention and Disaster containment. The Safety Information Model for the former provides the view about the compliance on constraints of a systems boundary and a safety practitioner could verify or correlate the details for measuring the practice compliance integrity. The Disaster Containment module post incident is used to aggregate the safety information and present a situational awareness view for the containment personnel's including the incident
The goals of Safety management Compliance are depicted below.

Upon these goals, the observables are presented that categorize into three types of categories, i.e. the Key Performance Indicators, Asset Design \& Construction, and the last on the periodic compliance. The basis of Safety management is built on Periodic Proof Testing to measure the integrity of the system. The information model map in Fig. 5., Fig. 6., Fig. 7., depicts the three attributes. The Labels (Process, Data, or People) hanging below an item classifies the category of the element. commanders.

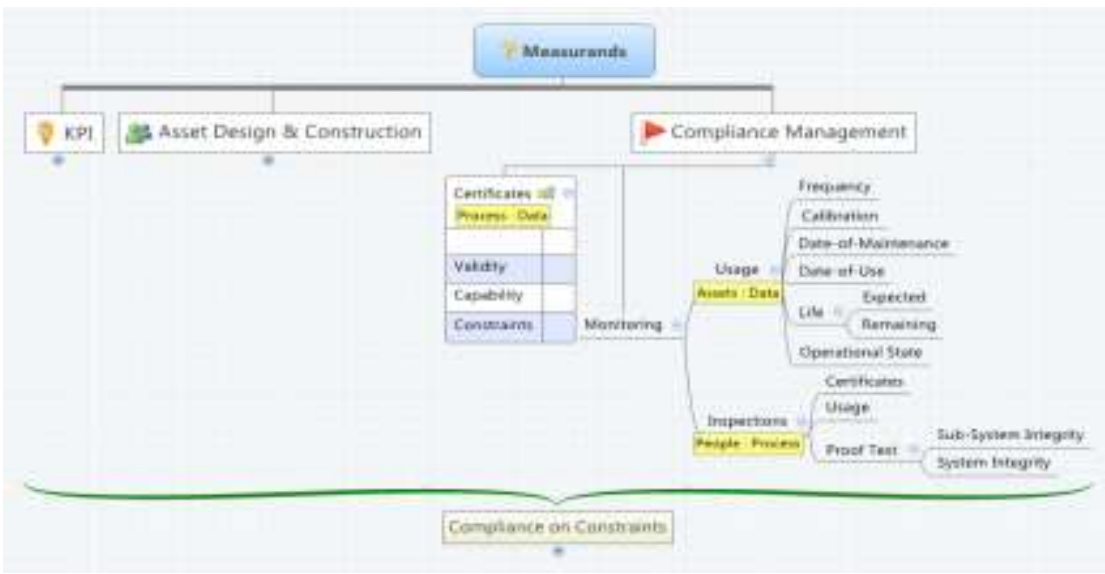

Fig. 5. Compliance Management 


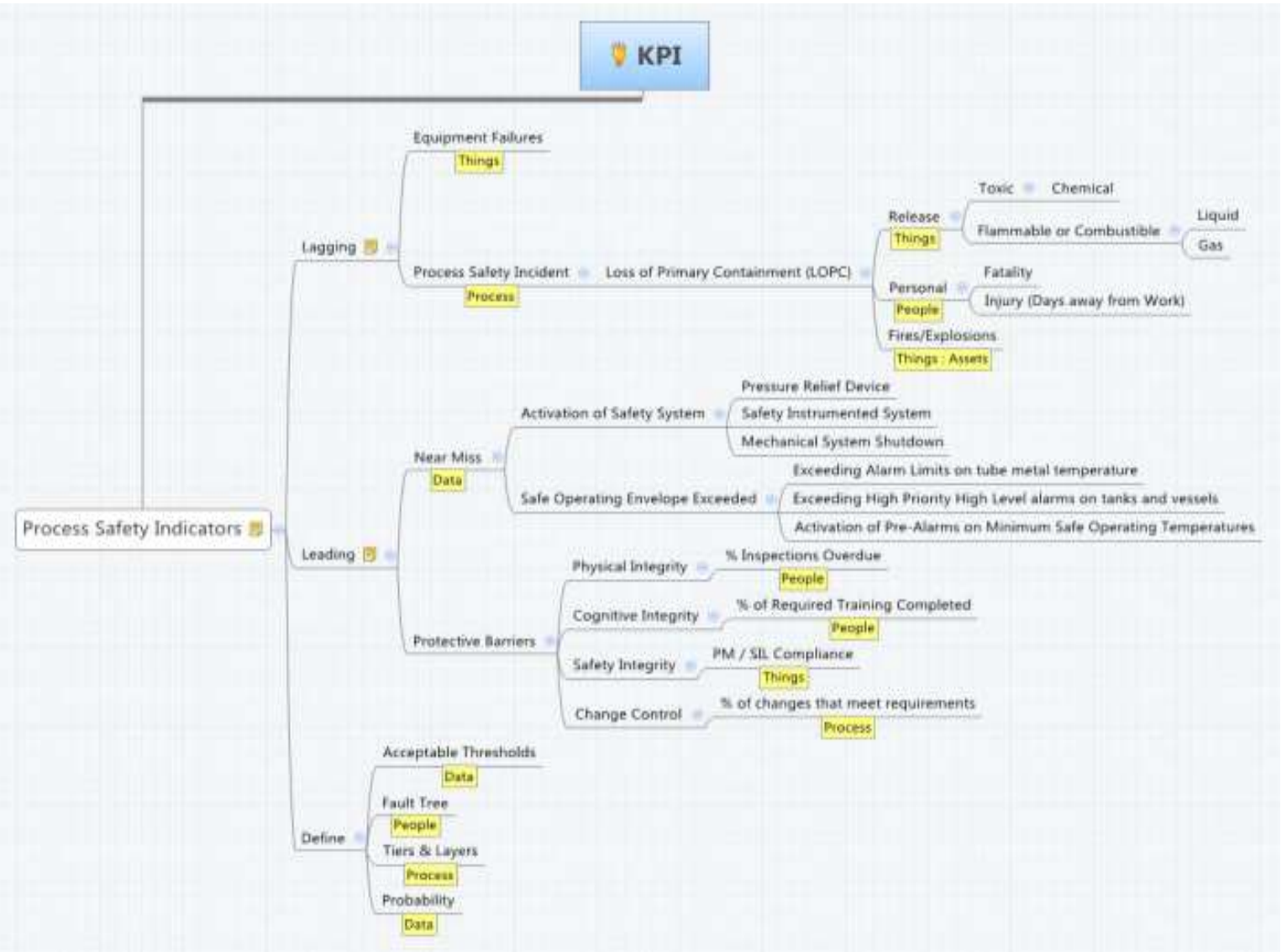

Fig. 6. Key Performance Indicators

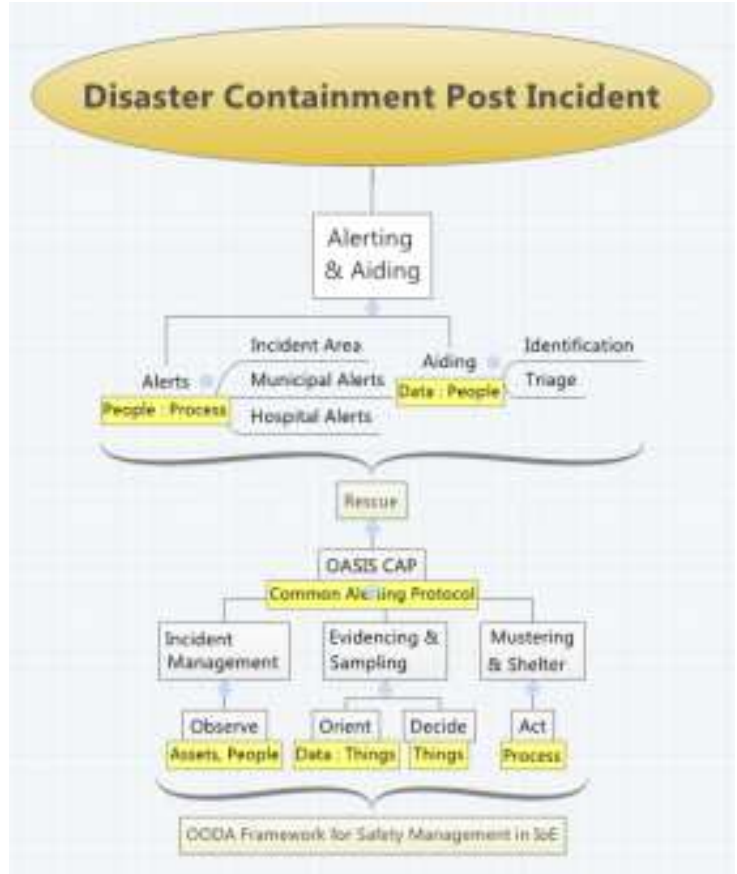

Fig. 7. Disaster Containment OODA Framework

The disaster containment view provides a view in which the Alert gets transported meaningfully to the respective recipients. The Department of Defense, USA, uses information presentation frameworks like Cursor on the Target(CoT) which is derived from the ObserveOrient-Decide- Act(OODA) framework for situational awareness [10].
The OASIS group for open information exchange has developed a standardized protocol for communicating critical alerts called the Common Alerting Protocol [11] which is now in version 1.2. This protocol has also been standardized by the International Telecommunications Union. The normal OODA framework, does not seek to obtain evidences as it is tied to the strict decision-action chain. This enhancement of adding the Evidencing module to the classical OODA helps in incident control and post incident analysis. This paper shall not further discuss about E-OODA in the current context.

\section{DISCUSSION}

The information models, along with the IoE label classification representation shows the different facets of the relationship of the data that exist among the three categories in the Safety management. A pivot table was used to order the nominal relationship among the three elements viz. (People, Process and Things) and KPI, Asset design, and compliance management. In Table 3 the types of People, Process and Things are described. The "things" are either Smart Tags or Smart Sensors. The connectivity process is described as Rules - If this then that, Verify - manual verification procedures, measure - a method or system to measure, and simulate - conditions are artificially injected and simulated. The people in the entire chain are either associated or informed or people acknowledge the measurements or process and are consciously aware. 
Table 3. IoE Classification

\begin{tabular}{|c|c|c|}
\hline People & Process & Things \\
\hline Inform $(I):$ are Informed & Rules $(R):$ are available for processing & $\begin{array}{c}\text { Static Smart Tag }(T): \text { communicating tags } \\
\text { with Reference Constant readings embedded inside. }\end{array}$ \\
\hline Associate $(A):$ take passive part in the role. & Verify $(V):$ people are expected to verify & Smart Sense $(M)$ : communicating smart sensors. \\
\hline $\begin{array}{c}\text { acKnowledge }(K): \text { take active role in } \\
\text { the process and are consciously aware. }\end{array}$ & $\begin{array}{c}\text { Measure }(M): \text { system measurements are made. } \\
\text { Simulate }(S): \text { conditions are simulated to } \\
\text { verify system behavior }\end{array}$ & \\
\hline
\end{tabular}

Table 4. IoE in Asset Design \& Construction

\begin{tabular}{|c|c|c|c|}
\hline \multicolumn{4}{|c|}{ Asset Design \& Construction } \\
\hline Process & Things & People & Category \\
\hline \multirow{13}{*}{ Rules } & \multirow{5}{*}{ Tag } & \multirow{4}{*}{ acK } & o Permissible Exposure Limit \\
\hline & & & o Storage \\
\hline & & & o Max. Inventory \\
\hline & & & o Toxicity \\
\hline & & Associate & o Construction, Electrical \\
\hline & \multirow{8}{*}{ senseM } & \multirow{7}{*}{$\mathrm{acK}$} & $\S$ - Capacity \\
\hline & & & $\S$ - Composition \\
\hline & & & $\S-$ Flow \\
\hline & & & $\S$ - Pressure \\
\hline & & & $\S$-Temperature \\
\hline & & & o hazardous effect \\
\hline & & & o Stability \\
\hline & & Inform & $\begin{array}{l}\text { Inventory Levels for } \\
\text { Process Chemicals }\end{array}$ \\
\hline Simulate & senseM & Inform & o Consequences \\
\hline \multirow{8}{*}{ Verify } & \multirow{7}{*}{ Tag } & \multirow{4}{*}{$\mathrm{acK}$} & o Design Codes \\
\hline & & & o Process Chemistry \\
\hline & & & o Process Control System \\
\hline & & & o Safe Operating Range \\
\hline & & \multirow{3}{*}{ Inform } & o Corrosiveness \\
\hline & & & o Reactivity \\
\hline & & & o Process Flow Diagrams \\
\hline & senseM & Associate & o Inlet, Outlet \\
\hline
\end{tabular}

Table 4., represents relationship between Design \& IOE. The process here describes a system or system component in which the connected system behavior executes.

During Asset Design \& Construction, a smart tag is placed that defines the overall Permissible Exposure Limit in the defined area. There exists a rule in the system that checks for the exceptions to the permissible exposure limits. There is then a super rule that checks for the exception of either non-availability of rules or absence of tags for authorities to enforce. Similarly accidents are caused predominantly by extremities rather than the process itself. Thus uncontrolled or leaky inlets or outlets are cause of concern. Users are associated to such points and the sensors are used to measure for undesired behavior and such users shall verify the behavior periodically. There is then a super verify function that checks for user association and a rule to raise exceptions.
Table 5. IoE \& KPI

\begin{tabular}{|c|c|c|l|}
\hline \multicolumn{2}{|c|}{ KPI } \\
\hline Process & Things & People & \multicolumn{1}{c|}{ Category } \\
\hline Measure & senseM & acK & o Equipment Failures \\
\hline & & Inform & $\S$ Chemical Release \\
\hline Rules & senseM & Inform & $\S$ Change Control \\
\hline & & & $\S$ Cognitive Integrity \\
\hline & & & $\S$ Physical Integrity \\
\hline Verify & senseM & Associate & $\S$ Personnel Injury \\
\hline & & Inform & o Near Misses \\
\hline & Tag & acK & o Fault Tree \\
\hline & & & $\begin{array}{l}\text { o Normally } \\
\text { Accepted Thresholds }\end{array}$ \\
\hline & & & $\begin{array}{l}\text { o Probabilistic } \\
\text { Failure Modes \& SIL Level }\end{array}$ \\
\hline
\end{tabular}

Table 6. IoE \& Compliance Metrics

\begin{tabular}{|c|c|c|c|}
\hline \multicolumn{4}{|c|}{ Compliance Metrics } \\
\hline Process & Things & People & Category \\
\hline \multirow[t]{5}{*}{ Measure } & senseM & $\mathrm{acK}$ & $\begin{array}{l}\S \text { Proof Test - } \\
\text { Sub System Integrity }\end{array}$ \\
\hline & & & $\S$ Proof Test - System Integrity \\
\hline & & Associate & $\S$ Frequency \\
\hline & & & Associate \\
\hline & & Inform & $\S$ Remaining Life \\
\hline \multirow[t]{5}{*}{ Rules } & Tag & $\mathrm{acK}$ & $\S$ Certificates \\
\hline & & & $\S$ Usage \\
\hline & & & o Capability \\
\hline & & Inform & $\S$ Expected Life \\
\hline & & & o Validity \\
\hline \multirow[t]{4}{*}{ Verify } & Tag & $\mathrm{acK}$ & $\S$ Last Date of Maintenance \\
\hline & & & $\S$ Last Date of Use \\
\hline & & & $\S$ Operational State \\
\hline & & & o Constraints \\
\hline
\end{tabular}

Table 5., represents relationship in KPI and IoE. Table 6., represents relationship between Compliance Management \& IOE.

As discussed earlier, in the compliance management scenario, the user is associated to the proof testing process and acknowledges the behaviors and is recorded by a sense(Measure) process. There is then a Super 
Measure function to assimilate the over-all proof test observations to calculate system integrity. Smart tags are used to place certificate credentials and rules are placed to manage exceptions. The user plays a consciously aware role to acknowledge the compliance validity. There is then a super rule to measure compliance invalidation as above and raise exceptions.

Dr. Sam Mannan, in his statement to the US Senate on the City of West, Texas accident 2013 highlights the need for certifying $3^{\text {rd }}$ party agencies to assist OSHA and DHS in periodic inspection and verifying the authenticity and integrity of the plants or facilities to avoid such further catastrophes [12].The broad-banding work of the public safety telecom networks presents as a boon to use the computing powers to help the first-responders and civic authorities to do mandated compliance checks and as well plan well for emergencies.

The following figures(Fig. 8., Fig. 9., Fig. 10) depicts one set of information representation view for compliance management.

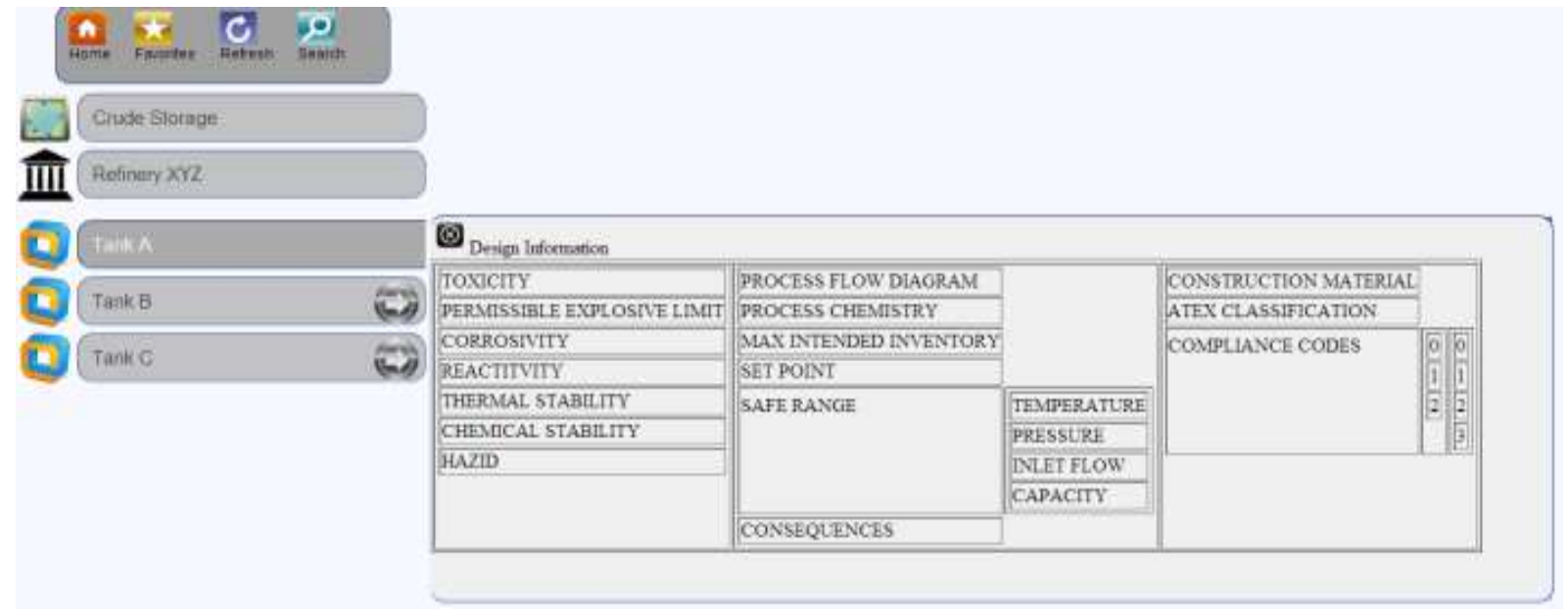

Fig. 8. SIM Compliance Information View
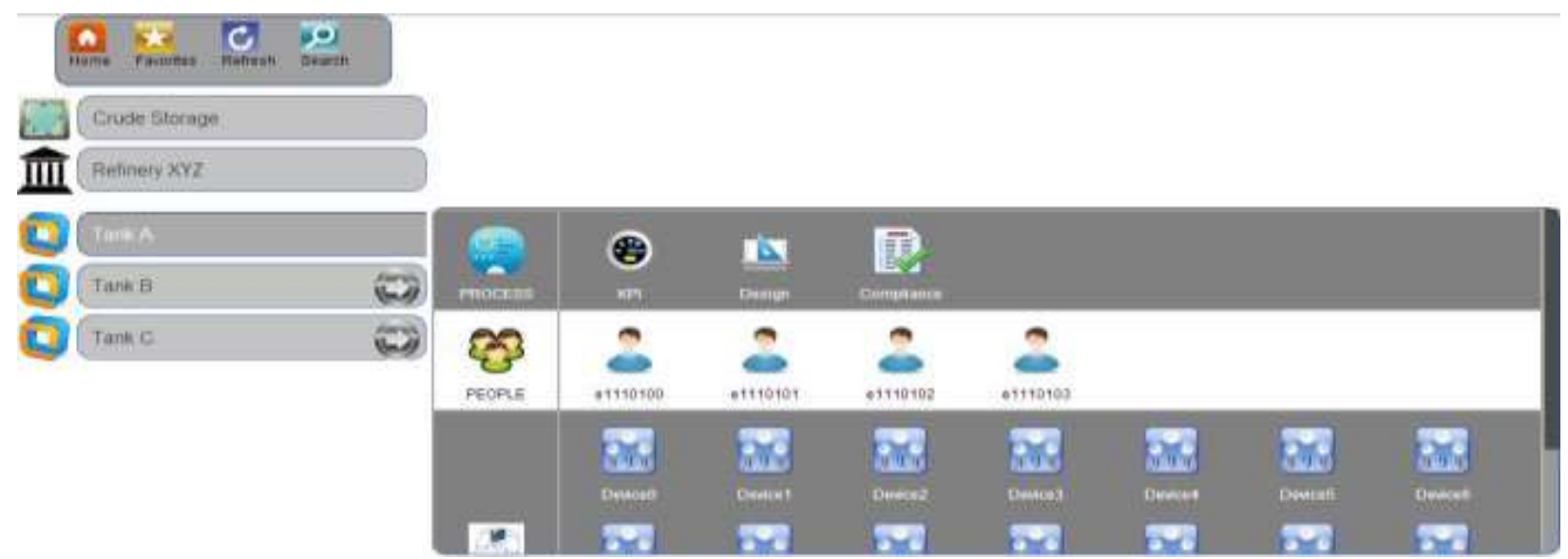

Fig. 9. Safety Information Model IoE Realization

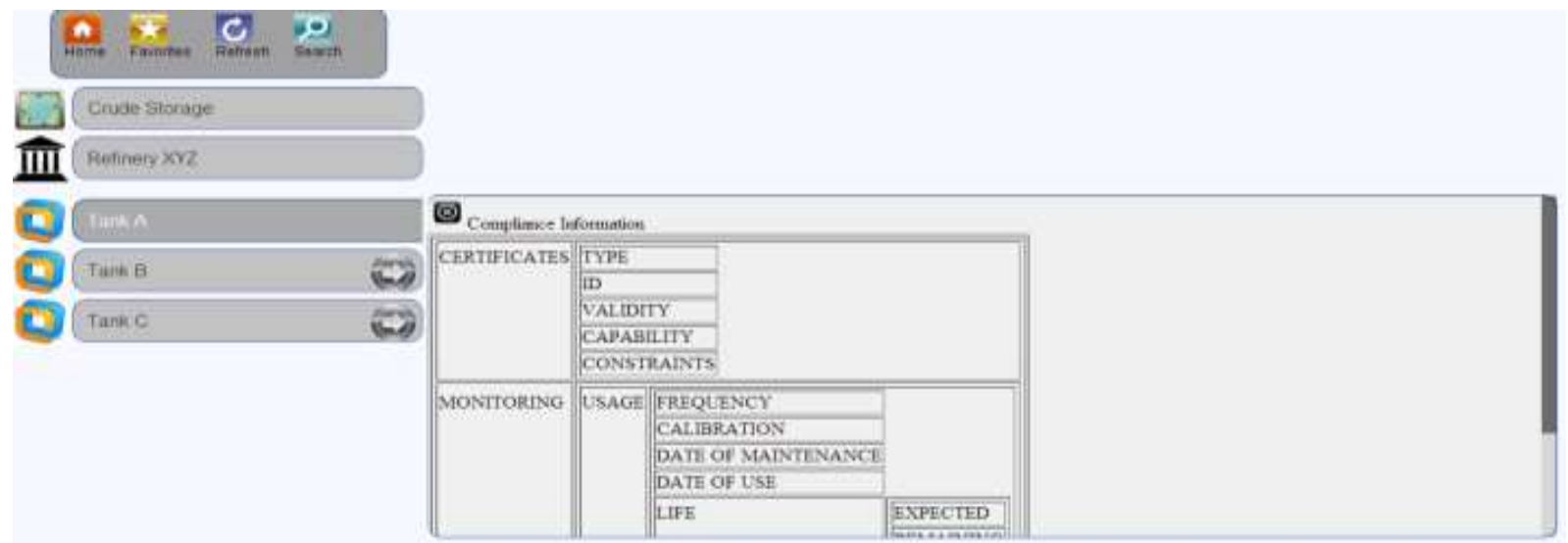

Fig. 10. SIM Design Information Details 
As depicted in Fig. 9. Safety Information Model IoE Realization, plant information can be viewed as the detail list of assets inside the plant segregated by different geographical areas and each one of the assets such as the tanks, effluent pipes or distillation units can have associated users, process and the devices within the system. In a compliance conformance view the safety incharge or the federal user can use the user-interface element for recording or observing the safety information.

In the case of City of West Fertilizer company accident, if the compliance information was recorded regularly, the mismatch in the designed tank storage content could have been identified and necessary actions could have been taken. The advancement of sensor networks and internet of everything could help in increasing compliance and averting such future accidents.

In the aftermath of an incidence i.e. a disaster the operational view of this public safety dashboard could include information from the medical agencies, first responders and also the legal compliance for post incident investigations.

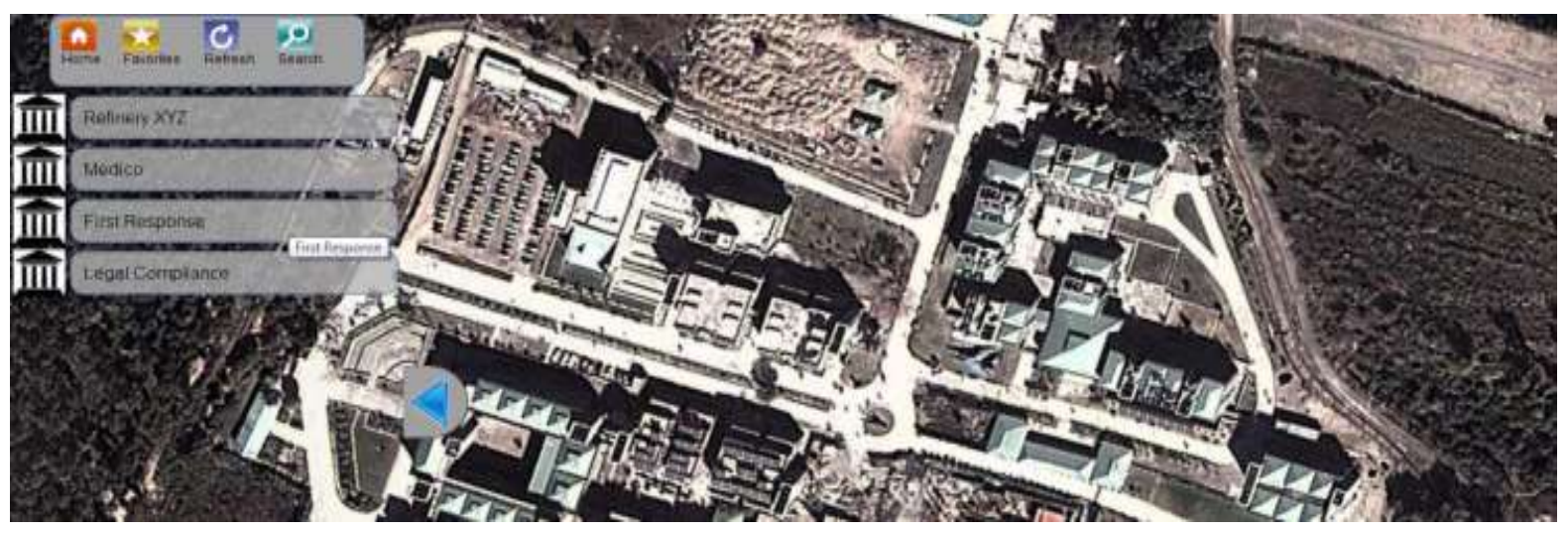

Fig. 11. Public Safety Common Operating Picture

\section{CONCLUSION}

The setup of Building Information Model (BIM) has helped the building management and construction safety industry with tools to design, manage and protect the facilities. Similarly a Safety information model, coupled with Industrial / federal compliance management solutions would aid in achieving cumulative public safety. As Santos-Reyes \& Beard [4] hope that a Systemic Safety Management System (SSMS) would be a potentially preventive management system, this information model studies the different use cases of construction, compliance adherence, key performance indicators for preventive/compliant safety management system. The OODA attributes required for managing an aftermath are also discussed and an enhancement is proposed specifically for safety management system. This method of information segregation and aggregation helps in providing higher order compliance and an overall safety. This information model also seconds and aids Sam Mannan's report on the City of West, Texas accident signifies the need for effective information management and periodic compliance audits required in the industry to avert such accidents happening in the future.

\section{REFERENCES}

[1] O. Vermesan and P. Friess, Internet of Things-Global Technological and Societal Trends From Smart Environments and Spaces to Green ICT, River Publishers, 2011.
[2] T. P. Kelly, Arguing safety: a systematic approach to managing safety cases, University of York, 1999.

[3] S. Zhang, J. Teizer, J.-K. Lee, C. M. Eastman and M. Venugopal, "Building information modeling (BIM) and safety: automatic safety checking of construction models and schedules," Automation in Construction, vol. 29, pp. 183--195, 2013.

[4] J. Santos-Reyes and A. N. Beard, "A systemic approach to managing safety," Journal of Loss Prevention in the process industries, vol. 21, pp. 15-28, 2008.

[5] Dept. of Homeland Security, "Public Safety Architecture Framework Volume I, II, III," Department of Homeland Security, 2006.

[6] K. Balachandran, K. Budka, T. Chu, T. Doumi and J. Kang, "Mobile responder communication networks for public safety," Communications Magazine, IEEE, 2006.

[7] Edge Innovation Network, "Edge Innovation Center of Emergency Informatics," Sept 2013. [Online]. Available: http://www.edge-innovation.com/.

[8] Wikipedia, "Wikipedia Safety Management Systems," Oct 2013. [Online]. Available: http://en.wikipedia.org/wiki/Safety_management_syste ms.

[9] "Capsnet Strategic Plan," California Technology Agency, 0303 2011. [Online]. Available: http://www.caloes.ca.gov/PSC/Documents/PDF/CAPS NET_Strategic_Plan_03-03-2011.pdf. [Accessed 10 10 2012].

[10] J. R. Boyd, "Organic design for command and control," A discourse on winning and losing, 1987. 
[11] OASIS Open, "Common Alerting Protocol," 1 July 2010. [Online]. Available: http://docs.oasisopen.org/emergency/cap/v1.2/CAP-v1.2-os.html.

[12] D. S. Mannan, "Environment and Public Works," Mary Kay O'Connor Process Safety Center Texas A\&M Engineering Experiment Station, 27 June 2013. [Online]. Available: http://www.epw.senate.gov/public/index.cfm?FuseActi on=Files.View\&FileStore_id=12b33b05-57d8-474aa5d2-ded91814b20c. [Accessed 29 June 2013].

[13] 3G Partnership Program, "3GPP Public Safety," July 2013. [Online]. Available: www.3gpp.org/publicsafety.

[14] "Public Safety Architecture Framework Volume I, II, III," 2006.

[15] D. Evans, "The Internet of Everything: How More Relevant and Valuable Connections Will Change the World," CISCO White Paper [Online]. http://www. cisco. com/web/about/ac79/docs/innov/IoE. pdf, last access: July, 2013.

[16] N.-T. Dinh and Y. Kim, "RESTful Architecture of Wireless Sensor Network for Building Management System.," KSII Transactions on Internet \& Information Systems, vol. 6, 2012.

[17] D. W. B. a. D. C. Lawrence, "Principles of Emergency Management," 2007.

\section{Authors' Profiles \\ 1) $S$ B Aanandh}

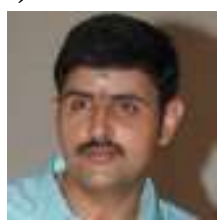

Aanandh completed his bachelor's in electronics and communication engineering from Thiagarajar College of Engineering, Madurai. He is a Certified Functional Safety Engineer for HW \& SW Engineering from TUV Rhineland and is pursuing a $\mathrm{PhD}$ in Publics Safety Communication Systems Design with University of Petroleum and Energy Studies Dehradun. Aanandh is currently employed with Honeywell Technology Solutions as the Leader for Core Architecture and Reuse Engineering for Automation \& Control Solutions, where he leads the initiatives on relevant core architectures, architectural evaluations and systematic design for reuse.

Aanandh has authored nearly 25 invention disclosures in the areas of Life Safety, Critical Infrastructure Protection and Situational Awareness. Aanandh has published 3 conference / journal papers in Chaotic Cryptography, and Measurement Instrumentation for Yarn Quality Testing. In his current $\mathrm{PhD}$ work he has published his review of Safety Models and concept of Safety Grid in International Journal of System Safety. Aanandh is also the recipient of Honeywell's Global Technical Excellence Award.

\section{2) Chinmaya Kar}

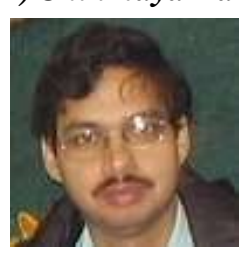

Dr. Chinmaya Kar completed bachelors in mechanical engineering, masters in Industrial engineering and management and $\mathrm{Ph} . \mathrm{D}$. in mechanical engineering. $\mathrm{He}$ has 11 years of research experience at various organizations such as Honeywell ACS Advanced Technology Lab, General Electric Global Research, INSA (Lyon, France), Crompton Greaves Limited and IIT Kharagpur. He has also 7 years teaching experience. His interests include reliability analysis and condition monitoring, data analytics (such as decision support system, signal processing, statistics etc.), and internet of everything. Currently, he is a Honeywell Fellow at Honeywell Technology Solutions, where he is leading initiatives of 'Condition Monitoring' and 'Internet of Everything'. Chinmaya is part of various initiatives on products/ prototypes such as Asset Manager, Equipment Health Monitoring, Idler Monitoring, Compressor Blade Health Monitoring etc. He is leading global projects such as WiBRATE (under European Commission's FP7 grant) and Idler Monitoring under these initiatives. Chinmaya has authored nearly 20 publications in various journals and conferences, 13 patents, 2 provisional patents, 3 trade secrets and nearly 36 disclosures (reports). He is a recipient of Erasmus Mundus Fellowship from European Commission and High-Value Ph.D. Fellowship from IIT, Kharagpur. He has also received several awards from Honeywell and GE - notably the annual Prolific Innovator award from Honeywell in 2012 and Management award from $\mathrm{GE}$ in 2007. He is a reviewer of journals such as 'IEEE Transaction on Industrial Electronics', 'Mechanical Systems and Signal processing', and 'Journal of Sound and Vibration' and 'Journal of Vibration and Control'. He has chaired sessions at International conference of CM/MFPT 2013 held at Poland. $\mathrm{He}$ is a certified vibration analyst Cat III and has certification on six sigma DFSS greenbelt. He has delivered a number of invited talks at different organizations.

\section{3) Nihal Siddiqui}

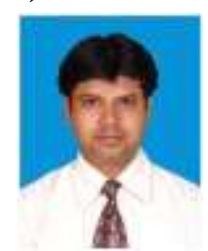

Dr. Siddiqui did his post graduation in Environmental Science and Doctorate in Environmental Biology. Dr Siddiqui specializes in the area of Environmental Pollution, Env. Monitoring \& control techniques and Disaster Management. $\mathrm{He}$ is currently associated with University of Petroleum \& Energy Studies, Dehradun as the head of health safety \& environmental engineering department. Dr. Siddiqui was also associated with Health, Safety \& Environment dept of ICEM college, Muscat , Oman University of Central Lancashire, UK. He has more than 65 Research papers to his credit and has participated in several National and International conferences. Dr. Siddiqui has authored 2 books viz. Natural Resources \& Environmental Management \& Handbook on Fire $\&$ safety. Dr. Siddiqui has guided more than 50 M.Tech and 7 $\mathrm{PhD}$ theses.

How to cite this paper: S B Aanandh, Chinmaya Kar, Nihal Siddiqqui,"Safety Information Modeling: Smart Safety Devices \& Internet of Everything", International Journal of Intelligent Systems and Applications (IJISA), vol.7, no.2, pp.41-49, 2015. DOI: $10.5815 /$ ijisa.2015.02.06 